Article

\title{
In Vivo Acute on Chronic Ethanol Effects in Liver: A Mouse Model Exhibiting Exacerbated Injury, Altered Metabolic and Epigenetic Responses
}

\author{
Shivendra D. Shukla ${ }^{1, *}$, Annayya R. Aroor ${ }^{1}$, Ricardo Restrepo ${ }^{1}$, Kusum K. Kharbanda ${ }^{2}$ and \\ Jamal A. Ibdah ${ }^{3}$
}

1 Department of Medical Pharmacology and Physiology, School of Medicine, University of Missouri, Columbia, MI 65212, USA; E-Mails: aroora@health.missouri.edu (A.R.A.); restreporj@health.missouri.edu (R.R.)

2 Veterans Affairs Nebraska-Western Iowa Health Care System, Omaha, NE 68105, USA; E-Mail: Kusum.Kharbanda@va.gov

3 Department of Internal Medicine, School of Medicine, University of Missouri, Columbia, MI 65212, USA; E-Mail: ibdahj@health.missouri.edu

* Author to whom correspondence should be addressed; E-Mail: shuklaSD@missouri.edu; Tel.: +1-573-882-2740; Fax: +1-573-884-4558.

Academic Editors: Natalia Osna and Kusum Kharbanda

Received: 13 August 2015 / Accepted: 17 November 2015 / Published: 20 November 2015

\begin{abstract}
Chronic alcoholics who also binge drink (i.e., acute on chronic) are prone to an exacerbated liver injury but its mechanism is not understood. We therefore investigated the in vivo effects of chronic and binge ethanol ingestion and compared to chronic ethanol followed by three repeat binge ethanol on the liver of male C57/BL6 mice fed ethanol in liquid diet (4\%) for four weeks followed by binge ethanol (intragastric administration, $3.5 \mathrm{~g} / \mathrm{kg}$ body weight, three doses, $12 \mathrm{~h}$ apart). Chronic followed by binge ethanol exacerbated fat accumulation, necrosis, decrease in hepatic SAM and SAM:SAH ratio, increase in adenosine levels, and elevated CYP2E1 levels. Histone H3 lysine acetylation (H3AcK9), dually modified phosphoacetylated histone H3 (H3AcK9/PS10), and phosphorylated H2AX increased after binge whereas phosphorylation of histone $\mathrm{H} 3$ ser 10 (H3S10) and H3 ser 28 (H3S28) increased after chronic ethanol-binge. Histone H3 lysine 4 and 9 dimethylation increased with a marked dimethylation in $\mathrm{H} 3 \mathrm{~K} 9$ in chronic ethanol binge group. Trimethylated histone H3 levels did not change. Nuclear levels of histone acetyl transferase GCN5 and histone deacetylase HDAC3 were elevated whereas phospho-CREB decreased in a distinctive
\end{abstract}


manner. Taken together, acute on chronic ethanol ingestion caused amplification of liver injury and elicited characteristic profiles of histone modifications, metabolic alterations, and changes in nuclear protein levels. These findings demonstrate that chronic ethanol exposure renders liver more susceptible to repeat acute/binge ethanol induced acceleration of alcoholic liver disease.

Keywords: acute ethanol; acute on chronic ethanol; alcoholic liver disease; binge alcohol; chronic-binge alcohol; epigenetics; histone modifications; mouse liver

\section{Introduction}

Binge drinking associated with chronic alcohol consumption is a major public health concern in the United States and around the globe [1-4]. Binge drinking in adolescent and young adult populations is rising at alarming proportions [5,6]. Most of the patients admitted to hospital with liver disease are alcoholics with binge drinking patterns [2-4]. Heavy binge drinking by chronic alcohol abusers is the trigger for augmenting liver injury [7-9] but the molecular mechanism sensitizing the progression of liver injury by binge after chronic ethanol intake is poorly understood.

Oxidative stress, increase in adenosine levels, and dysregulated methionine metabolism are considered to be contributing factors for the progression of liver injury [10-13] and the role of epigenetic mechanisms mediating progression of alcoholic liver injury is increasingly being recognized [14,15]. Among the epigenetic factors regulating gene expression, differential pattern of epigenetic histone modifications also determine activation of different sets of genes. Stable epigenetic histone modifications impact long term and transgenerational effects on gene expression but dynamic changes contribute to short term or prolonged effects on gene expression [16,17]. Epigenetic histone modifications are implicated in liver injury by binge [14,15,18-24], chronic alcohol consumption [25-28], and chronic alcohol administration followed by binge $[29,30]$.

We have shown in a clinically relevant rat model that chronic ethanol followed by repeat ethanol binge is accompanied by augmentation of liver injury [30]. In the present study, we have utilized mice model to examine the effects and potential mechanisms underlying alcohol induced liver injury [31,32].

\section{Results}

\subsection{Increased Hepatic Steatosis, Necrosis, Adenosine, CYP2E Levels and Decreased $S$-Adenosylmethionine by Binge Administration in Chronic Ethanol Treated Mice}

Chronic ethanol administration or acute repeat binge caused only a moderate increase in liver steatosis, which was mainly microvascular, whereas there was a marked macrosteatosis in the chronic-binge ethanol group (Figure 1A). Liver necrosis, as determined by measurement of ALT levels, increased modestly above control in chronic ethanol but was statistically insignificant. In chronic ethanol followed by binge treatment, ALT increased significantly by $275 \%$ above control (Figure 1B). Triglyceride levels increased in all groups above control (Figure 1C). Compared to control group, the TG levels were 2, 4, and 13 folds higher in E, CB, and EB groups, respectively. Noticeably, the highest TG levels were in 
chronic ethanol-binge (EB) group that was about four fold higher than binge alone group (CB), and about seven fold higher than the chronic ethanol (E) group. Chronic ethanol increased CYP2E1 protein levels only modestly (Figure 1D) while binge ethanol generated a much higher CYP2E1 levels compared with controls. The largest increase (2.5 fold) was seen in the chronic ethanol-binge group (Figure 1D).

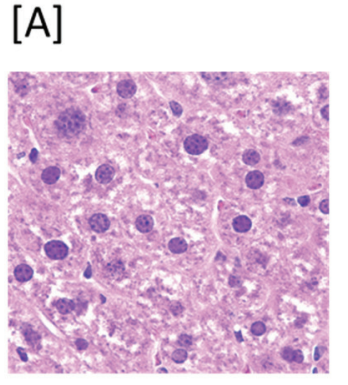

C

[B]

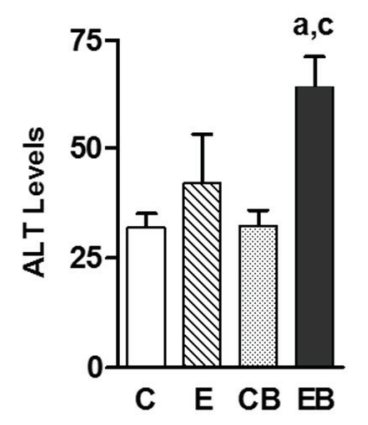

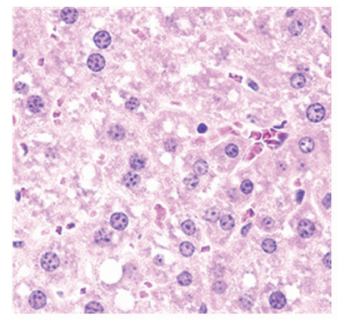

$\mathrm{E}$

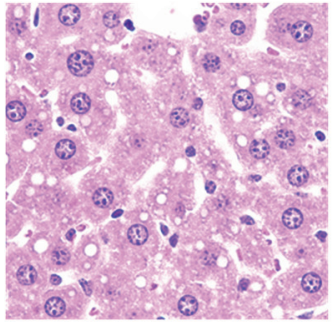

$\mathrm{CB}$

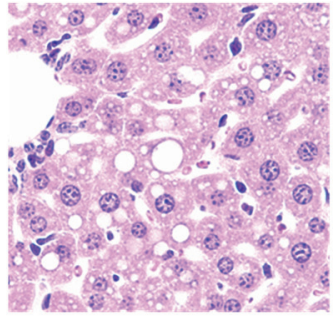

EB
[C]

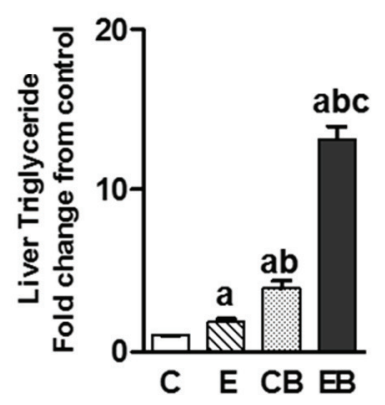

[D]

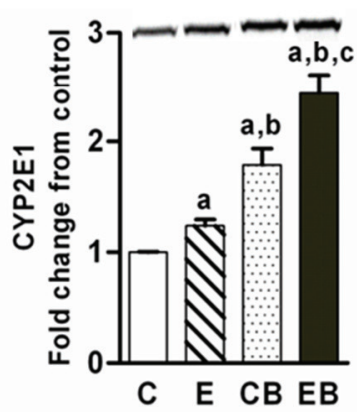

Figure 1. Hepatic steatosis, serum ALT, and CYP2E1 levels in chronic ethanol and binge treated mice. The chronic ethanol feeding (four weeks) and three repeat binge treatment was performed as described under "Experimental Section". (A) Hepatic steatosis: sections of liver samples were stained with hematoxylin and eosin; (B) Serum ALT levels; (C) Liver triglyceride levels; (D) CYP2E1 protein measured by western blotting (representative blot images shown above the histogram). Values are mean $\pm \mathrm{SE}(n=3$ to 4 mice). a: significant compared to control $(p<0.05)$; b: significant from chronic ethanol group $(p<0.05)$; c: significant compared to control-binge. C: Control (pair fed); E: Chronic ethanol; $\mathrm{CB}$ : Control ethanol binge; EB: Chronic-ethanol-binge.

Dysregulated methionine metabolism has been reported in chronic ethanol treated mice $[11,25]$. Therefore, we determined hepatic levels of $s$-adenosyl methionine (SAM) and $s$-adenosyl homocysteine (SAH). Under the experimental conditions used in the present study, chronic ethanol increased SAM levels but a subsequent binge in chronic ethanol treated group resulted in a decrease that was statistically significant (Figure 2A). SAH levels were not significantly different among chronic ethanol, binge, and chronic ethanol binge groups. In chronic followed by three binge (EB) samples there was an apparent decrease in SAM/SAH ratio as compared to Control (C), chronic ethanol (E), or control binge (CB) groups (Figure 2C). Chronic ethanol increased GSH and binge on chronic had no further effect on GSH levels (Figure 2D). Upregulation of adenosine signaling has been shown to contribute to chronic alcoholic liver injury [12]. 
Hence, hepatic adenosine concentration in chronic, binge and chronic binge ethanol group were evaluated (Figure 2E). Hepatic adenosine levels significantly decreased in chronic ethanol treated mice, but their levels increased by binge ethanol with the highest levels in chronic ethanol-binge liver (Figure 2E).

[A]

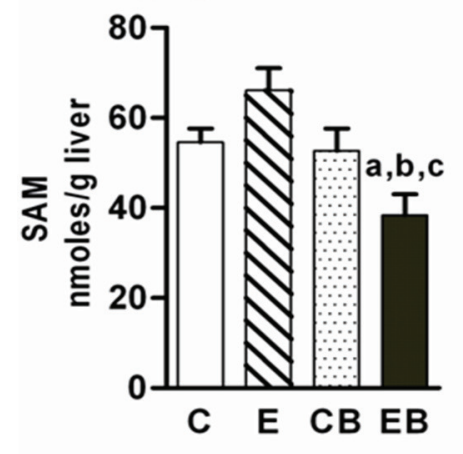

[D]

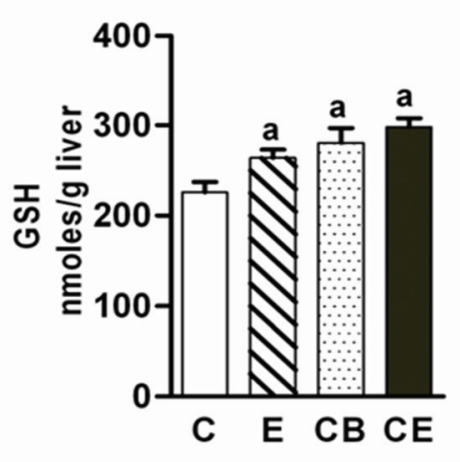

[B]

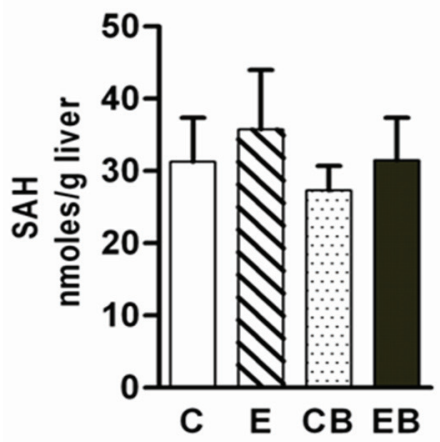

[E]

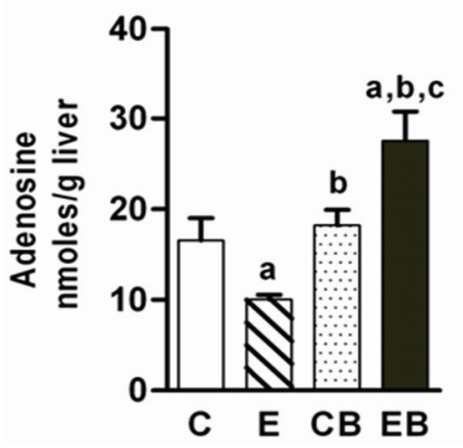

[C]

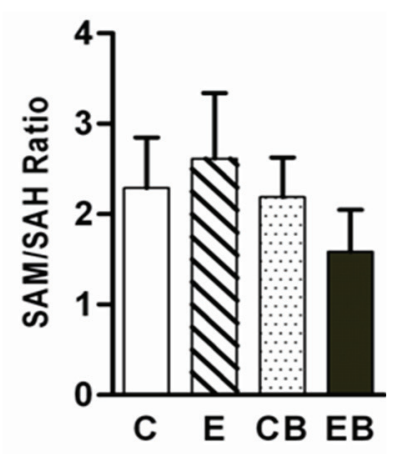

Figure 2. Hepatic $s$-adenosylmethionine (SAM), $s$-adenosyl homocysteine (SAH), adenosine and glutathione levels in chronic ethanol and binge treated mice. Experimental protocol was similar to that described in "Experimental Section". Values are mean \pm SE ( $n=4$ mice). a: significant compared to control $(p<0.05)$; b: significant from chronic ethanol group $(p<0.05)$; c: significant compared to control-binge. C: Control (pair fed); E: Chronic ethanol; CB: Control ethanol binge; EB: Chronic-ethanol-binge. (A) SAM; (B) SAH; (C) SAM/SAH ratio; (D) GSH; (E) Adenosine.

\subsection{Increased Phosphorylation of Histone H3 after Chronic Ethanol-Binge}

Post-translational modifications in histone proteins by ethanol have been shown earlier $[14,15]$. However, the acute on chronic ethanol influence on these modifications in mouse liver is not known and was therefore monitored. Increased phosphorylation of histone H3S10 (Figure 3A) and H3S28 (Figure 3B) indicate chromatin remodeling and gene transcription [14-17,23]. Phosphorylation of histone H3S10 (Figure 3A) and H3S28 (Figure 3B) did not change after chronic ethanol or binge administration alone whereas chronic ethanol followed by binge caused a significant increase in histone H3S10 and histone H3S28 phosphorylation (Figure 3A,B). 
[A]

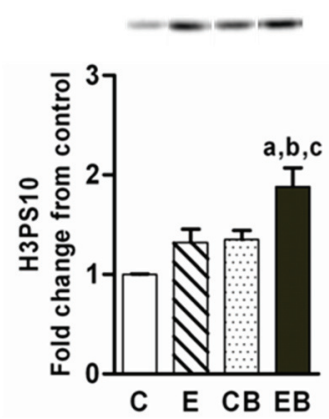

$[B]$

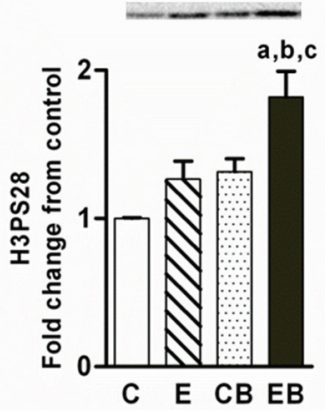

Figure 3. Phosphorylated histone H3S10 and S28 in chronic and chronic ethanol binge treated mice. The chronic ethanol feeding (four weeks) and three binge treatment was performed as described under "Experimental Section". The hepatic nuclear extracts were used for western blotting followed by quantitative imaging [30]. Images of representative blots are shown. Values are mean $\pm \mathrm{SE}(n=4$ mice). a: significant compared to control $(p<0.05)$; b: significant from chronic ethanol group $(p<0.05)$; $\mathrm{c}$ : significant compared to control-binge. C: Control (pair fed); E: Chronic ethanol; CB: Control ethanol binge; EB: Chronic-ethanol-binge. (A) H3PS10; (B) H3PS28.

\subsection{Levels of Dimethylated H3 K4, Dimethylated H3 K9, and Trimethylated H3K9}

Histone H3K4 methylation is implicated in transcriptional activation whereas histone H3K9 dimethylation and H3K9 trimethylation are involved in silencing of gene expression [15,17,18,25]. H3K4 dimethylation increased to similar levels in chronic ethanol, binge ethanol, and chronic ethanol-binge groups (Figure 4A). H3K9 dimethylation also increased in chronic ethanol, binge, and chronic ethanol binge group, but the extent of histone $\mathrm{H} 3 \mathrm{~K} 9$ dimethylation was more marked in chronic ethanol-binge group (Figure 4B). In contrast to above changes, the levels of trimethylated H3K9 remained unaltered in all the three groups (Figure 4C).

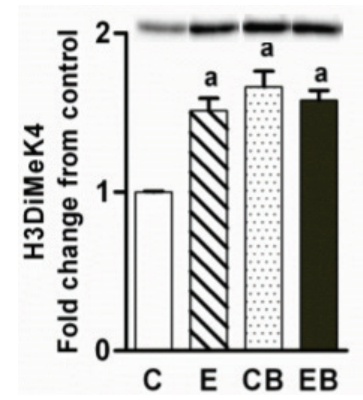

(A)

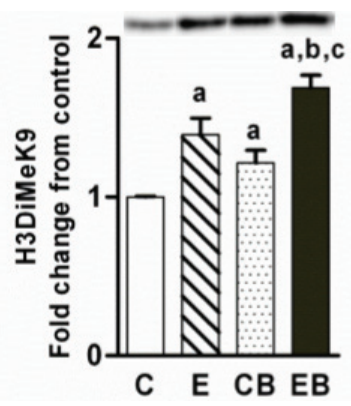

(B)

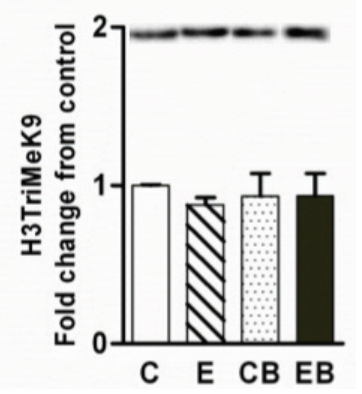

(C)

Figure 4. Levels of dimethylated $\mathrm{H} 3 \mathrm{~K} 4$, dimethylated histone $\mathrm{H} 3 \mathrm{~K} 9$, and trimethylated histone H3K9 in chronic ethanol and binge treated animals. The experimental protocol was as detailed in the "Experimental Section". The representative western blot images are also shown above the histograms. Values are mean $\pm \mathrm{SE}(n=4$ mice). a: significant compared to control $(p<0.05)$; $\mathrm{b}$ : significant from chronic ethanol group $(p<0.05)$; $\mathrm{c}$ : significant compared to control-binge. C: Control (pair fed); E: Chronic ethanol; CB: Control ethanol binge; EB: Chronic-ethanol-binge. (A) H3DiMeK4; (B) H3DiMeK9; (C) H3TriMeK9. 


\subsection{Increased H3K9 Acetylation, H3K9 Acetylation/S10 Phosphorylation and H2AX Phosphorylation}

Acetylation of histone $\mathrm{H} 3 \mathrm{~K} 9$ is one the significant epigenetic effects of ethanol in vitro and in vivo under the setting of binge and chronic ethanol binge in rats [15,30]. H3AcK9 levels did not increase after chronic ethanol intake (Figure 5A), increased after binge ethanol alone, and was not altered after chronic ethanol binge (Figure 5A). Histone H3K9 acetylation is often associated with accumulation of histone H3 dually modified by H3K9 acetylation/S10 phosphorylation (H3AcK9PS10) [15,22,23]. The levels of H3AcK9PS10 did not change under these treatment conditions except for a small increase in binge alone (Figure 5B). Histone $\mathrm{H} 2 \mathrm{AX}$ phosphorylation is implicated in nuclear DNA repair and is modulated by histone $\mathrm{H} 3$ acetylation [33-35]. Therefore, we examined H2AX phosphorylation in ethanol treated mice groups. H2AX phosphorylation showed a pattern similar to H3K9 acetylation with a significant increase in the acute/binge group (Figure 5C).

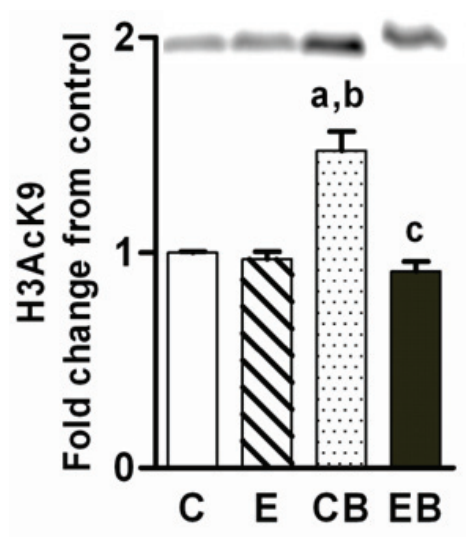

(A)

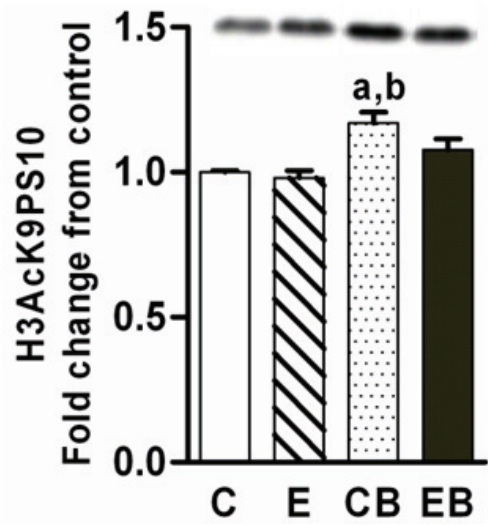

(B)

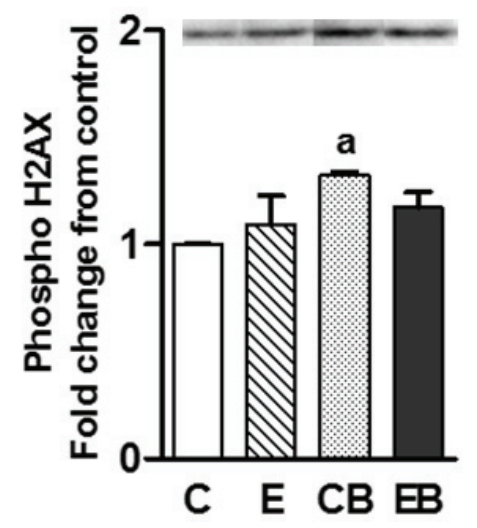

(C)

Figure 5. Levels of H3AcK9 (A); H3AcK9PS10 (B); and phospho-H2AX (C). The experimental details were as described in the "Experimental Section". The nuclear levels of these components were determined by western blotting (representative images are shown above the histograms). Values are mean $\pm \mathrm{SE}$ ( $n=4$ mice). a: significant compared to control $(p<0.05)$; b: significant from chronic ethanol group $(p<0.05)$. C: Control (pair fed); E: Chronic ethanol; $\mathrm{CB}$ : Control ethanol binge; EB: Chronic-ethanol binge.

\subsection{Increased Nuclear Levels of GCN5, HDAC3 and Decreased Levels of Phospho-CREB in Chronic} Ethanol, Binge and Chronic Ethanol-Binge Groups

Increased expression of HAT GCN5 and increased H3K9 acetylation by ethanol occurs in hepatoma cells [36]. Therefore we determined GCN5 protein levels in the liver nuclear extracts. GCN5 levels increased in chronic ethanol, binge, and chronic ethanol binge groups (Figure 6A) although H3K9 acetylation was significantly increased only in binge group (Figure 5A). It is not known if the differential pattern of GCN5 expression and H3K9 acetylation are due to altered levels of histone deacetylases. We therefore determined HDAC3 levels since HDAC3 levels are increased in acute ethanol administration in mice [21]. The HDAC3 protein levels in the nuclear extracts were increased in all three groups with maximum increase in the binge group but this increase was not statistically different from other groups (Figure 6B). 


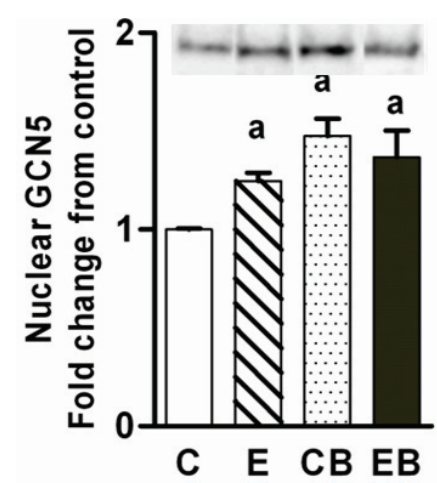

(A)

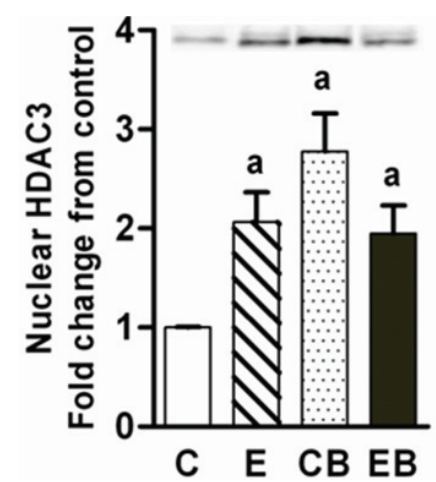

(B)

Figure 6. Levels of GCN5 (A); and HDAC 3 (B) in chronic ethanol-binge treated mice. The experimental protocol were as in the "Experimental Section". The levels of GCN5 and HDAC3 were determined by western blotting and the representative images of blots are shown above the histograms. Values are mean $\pm \mathrm{SE}(n=4$ mice). a: significant compared to control $(p<0.05)$. C: Control (pair fed); E: Chronic ethanol; CB: Control ethanol binge; EB: Chronic-ethanol-binge.

Phosphorylated CREB (cyclic AMP response element binding protein) has been shown to stimulate $\mathrm{CBP} / \mathrm{p} 300$ mediated acetylation of $\mathrm{H} 3 \mathrm{~K} 9$ and is one of the important transcriptional regulator of hepatic metabolic and inflammatory responses [35,37,38].

Phosphorylation of CREB that usually occurs in the cytosol facilitates its translocation into the nucleus [37,38]. Therefore, we determined the levels of phosphorylated CREB (P-CREB) and CREB protein in the whole cell (Figure 7A-C) and nuclear extracts (Figure 7D-F). In the whole cell extracts P-CREB increased in all the groups (Figure 7A) but the total CREB protein did not change in either of the groups (Figure 7B). However, there was a modest but statistically significant increase in P-CREB in binge (CB) and chronic ethanol-binge (EB) groups when compared to chronic ethanol (E) group (Figure 7A). The ratio of P-CREB/CREB was similar in all the three groups (Figure 7C). In contrast, in the nuclear fraction the levels of P-CREB gradually decreased in the following order: chronic $>$ binge alone $>$ chronic-binge (Figure 7D). The decrease in the binge group was more marked compared to chronic ethanol group but the most significant decrease occurred in the chronic ethanol-binge group. However, nuclear levels of CREB protein was not significantly different among the groups (Figure 7E) and changes in the ratio of P-CREB/CREB paralleled changes in nuclear levels of P-CREB (Figure 7F).

[A]

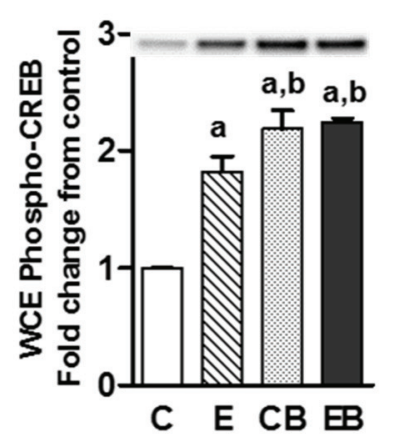

[B]

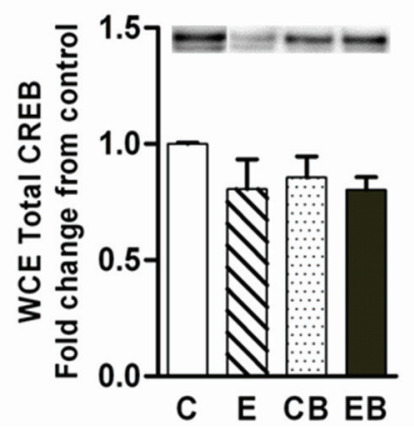

[C]

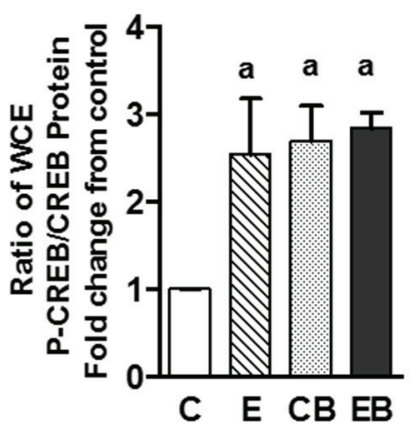

Figure 7. Cont. 
[D]

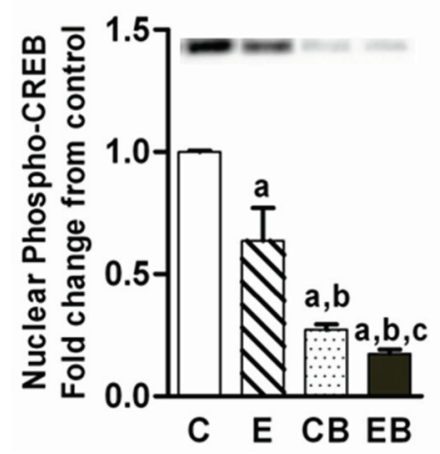

$[E]$

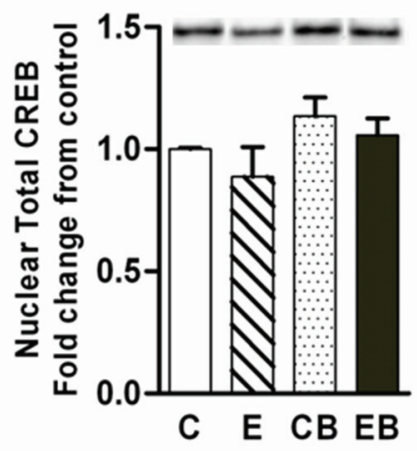

$[\mathrm{F}]$

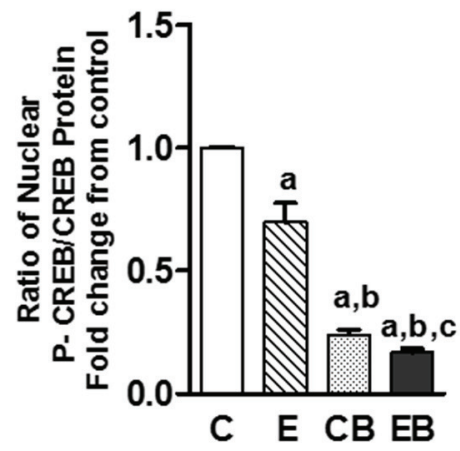

Figure 7. Phosphorylated CREB and CREB protein in hepatic extracts from chronic ethanol-binge treated mice. Changes in CREB and P-CREB were determined by western blotting of whole cells extracts (WCE, upper panel (A-C)); and nuclear fraction (lower panel (D-F)). Images of representative blots are also shown. The ratio of P-CREB/CREB are also shown. Values are mean $\pm \mathrm{SE}(n=4$ mice). a: significant compared to control $(p<0.05)$; b: significant from chronic ethanol group $(p<0.05)$; : significant compared to control-binge. C: Control (pair fed); E: Chronic ethanol; CB: Control ethanol binge; EB: Chronic-ethanol-binge.

\section{Discussion}

Heavy binge drinking in patients who are chronically consuming alcohol is the most common cause for the worsening of liver damage in chronic alcoholic liver disease [7-9]. To mimic this scenario we have developed an experimental animal model of chronic ethanol followed by repeat binge episode to examine augmentation of liver injury in mice. Earlier, we reported a rat model for chronic ethanol followed by three doses of acute binge administration [29,30]. In the rat model it was shown that 3 binge is more damaging than single binge in chronically treated animals [29]. In the present study, we have used a mouse model because both rat and mouse models serve as good experimental models to study alcoholic liver disease [32]. Moreover, the mouse model is useful for studying the progression of ALD due to increased susceptibility of mice for alcoholic liver injury and the feasibility of genetic manipulation in mice $[1,31,32]$. In this study, we explored for the first time, dysregulated methionine metabolism, adenosine accumulation and epigenetic histone modifications and enzymes related to histone modifications as determinates of augmentation of liver injury after binge ethanol administration in chronically ethanol treated mice. Although the pattern of injury was comparable to the rat model, there were differences in the magnitude of changes in biomarkers of liver injury. Additionally, the patterns of changes in histone modifications were distinct for the rat and mice models. Increased steatosis and elevated serum ALT levels were observed in the chronic ethanol binge group in this study and suggest exacerbation of chronic ethanol induced injury by binge administration. Ethanol causes increased levels of CYP2E1 in vivo and in primary hepatocytes thus contributing to ethanol induced oxidative stress [10,11,33]. Although CYP2E1 levels are increased after binge and chronic ethanol, the increase is most marked after chronic ethanol-binge suggesting an important role for oxidative stress in enhancing liver injury by acute on chronic ethanol ingestion. The role of CYP2E1 levels in the pathogenesis is 
underscored by the prevention of binge and chronic ethanol induced liver injury in CYP2E1 knock out mice $[10,31,32]$. Hepatic glutathione levels have been reported to be elevated, decreased, or unaltered by ethanol feeding $[39,40]$. In the present study, we observed that glutathione levels in whole liver extracts were not altered. In fact, the levels were mildly elevated in all three groups which could be a protective response of the liver to ethanol exposure.

Enhanced adenosine release from hepatic slices from alcohol treated mice and increased expression of adenosine receptors have been reported in animal models of alcoholic liver injury and in humans [12,13]. Moreover, adenosine receptor antagonists ameliorate liver injury in chronic alcohol treated mice [12,13]. The levels of hepatic adenosine were lower (compared to control) after chronic ethanol intake and remained unaltered after binge. However, its levels roughly doubled above control in chronic ethanol-binge liver. Therefore, increased levels of adenosine may contribute to the potentiation of chronic alcoholic liver injury by binge.

Dysregulation of methionine metabolism is one of the hepatotoxic effects of ethanol [11]. Hepatic $s$-adenosyl methionine (SAM) levels increased after chronic ethanol but decreased by chronic ethanol binge. Although increased SAM levels indicate adaptive response to chronic ethanol administration, the significant decrease in SAM levels and decreased ratio of SAM/SAH suggest increased cytotoxic effects of chronic ethanol-binge combination under the experimental protocols utilized in the present study. Decreases in SAM levels are associated with impairment in essential methylation reactions through inhibition of various methyltransferases including phosphatidylethanolamine methyltransferase, isoprenylcysteine carboxyl methyltransferase and protein L-isoaspartate methyltransferase. Decreased activity of these enzymes is associated with increased steatosis, increased apoptosis and increased accumulation of damaged proteins seen in alcoholic liver disease $[11,40]$.

Chronic ethanol intake or binge alone caused insignificant increase in histone H3S10 and H3S28 phosphorylation whereas binge after chronic ethanol increased histone H3S10 and H3S28 phosphorylation. In contrast to histone phosphorylation, histone $\mathrm{H} 3 \mathrm{~K} 4$ and $\mathrm{H} 3 \mathrm{~K} 9$ dimethylation increased in chronic, binge, and chronic binge groups. H3K4 dimethylation is usually associated with induction of gene expression and H3K9 dimethylation is associated with silencing of gene expression [15]. This suggests different sets of modified histone residues in chromatin remodeling complex with simultaneous upregulation and down regulation of different genes [18]. In this chronic-repeat binge mice model histone H3K9 trimethylation was not affected by ethanol. Moreover, increase in histone H3K4 and H3K9 dimethylation accompanied a decrease in SAM in chronic ethanol binge group suggesting histone $\mathrm{H} 3$ dimethylation by ethanol may occur independent of the availability of methyl donor and may involve other mechanisms. The cytokine tumor necrosis factor (TNF $\alpha$ ) and transcription factor early growth response gene (egr-1) are upregulated by histone H3 phosphorylation, and TNF $\alpha$ is silenced by histone H3K9 methylation $[23,41,42]$. Therefore, an increase in histone H3 phosphorylation in chronic ethanol-binge but not in chronic ethanol or binge suggests additional epigenetic modifications contributing to the amplification of liver injury. It is noteworthy that the changes in global histone modification are further fine-tuned by gene specific histone modifications resulting in multiple effects on gene expression as seen in chronic ethanol treated rats with unaltered global histone H3K9 acetylation but increased recruitment of histone $\mathrm{H} 3 \mathrm{~K} 9$ to the $\mathrm{ADH} 1$ promoter but not to c-jun, although expression of mRNA for both genes were increased [26]. 
In contrast to $\mathrm{H} 3 \mathrm{~S} 10$ and $\mathrm{H} 3 \mathrm{~S} 28$ phosphorylation, $\mathrm{H} 3 \mathrm{~K} 9$ acetylation was increased in the binge ethanol but not in the chronic ethanol-binge group. Histone H3K9 acetylation is dependent on a balance between the activation of histone acetyl transferases and histone deacetylases. We have reported a role of GCN5 in ethanol induced H3K9 acetylation in ethanol metabolizing human hepatoma cell line (VA13 cells) [36]. Levels of GCN5 increased in all the three groups although H3K9 acetylation occurred only after binge. HDAC3 levels also increased in all three groups. This may indicate a delicate balance between HAT and HDAC that is modulated by ethanol exposure. Dually modified H3AcK9/PS10 also increased by binge and suggests acetylation of phosphorylated histone H3. In this regard, HATs such as ATF2 histone acetyl transferase show preference for phosphorylated histones. Increased H3K9 acetylation is also accompanied by increased histone H2AX phosphorylation by binge. Role for GCN5 histone acetyl transferase in H3K9 acetylation associated with H2AX phosphorylation by SW1/SNF chromatin complex has been recently reported [34]. H2AX phosphorylation has been implicated in DNA repair [33,34]. However, this protective response is compromised when binge was administered to chronic ethanol treated mice. It is relevant to note that chronic ethanol binge also resulted in decreased levels of H2AX phosphorylation in rats [33].

CREB is a key transcription factor playing a pivotal role in liver regeneration, hepatic carbohydrate metabolism, suppression of hepatic lipogenesis, and hepatic inflammatory responses. The levels of phospho-CREB in the whole cell extract were significantly increased in chronic ethanol, binge and chronic ethanol binge but nuclear levels were markedly decreased with most pronounced decrease noted in the ethanol binge group. Thus, dysregulation of nuclear P-CREB levels may be an underlying mechanism in alcohol induced liver injury by both binge and chronic ethanol intake. One mechanism that may contribute to transcriptional regulation is $\mathrm{CREB}$ dependent $\mathrm{CBP} / \mathrm{p} 300$ mediated histone acetylation $[35,37]$.

\section{Experimental Section}

\subsection{Reagents}

Antibodies to histone H3AcK9, phospho-H3 ser 10, phospho-H3 ser 10, phospho-H3 ser 28, histone $\mathrm{H} 3$ protein, dimethyl H3-lys4, dimethyl H3-lys9, phopsho-H2AX were obtained from Chemicon (Temecula, CA, USA). Antibodies to GCN5 and CYP2E1 were procured form Santa Cruz (Dallas, TX, USA). Antibody to HDAC3, phospho-CREB, and CREB protein were procured from Cell signaling (Danvers, MA, USA). Triglyceride assay kit (Cat \#F6428), protease inhibitors cocktail (p8340), and anti $\beta$-actin antibody were purchased from Sigma-Aldrich (St. Louis, MO, USA).

\subsection{Experimental Design for Chronic Ethanol Feeding and Binge Administration}

Seven week old C57BL/6J male mice, were purchased from Harlan (Indianapolis, IN, USA) and housed under a 12-h/12-h light/dark cycle. Animals were permitted ad libitum consumption of standard laboratory chow for one week to acclimatize. This was followed by feeding animals with Lieber-DeCarli liquid diet (Dyets, Inc., Bethlehem, PA, USA; [29]). Ethanol was progressively introduced into the liquid diet starting at $1 \%(\mathrm{wt} / \mathrm{vol})$ for 2 days, increased to $2 \%$ for 2 days, $3 \%$ for 2 days, and $4 \%$ from day 7 onwards for 4 weeks. Pair-fed control mice were given a liquid diet in which ethanol was replaced by 
dextrin/maltose to maintain the isocaloric intake in the two groups. At the end of 4 weeks of feeding, mice were divided into four groups: Control group (C) received water, chronic ethanol group (E) received water, control-binge group (CB) received three doses of ethanol, and chronic ethanol-binge group (EB) received three doses of ethanol. The three doses of binge were administered intragastrically (3.5 gm/kg body weight) after diluting ethanol to $32 \%(v / v)$ in sterile water and orally gavaged to the stomach using a 20-gauge stainless steel blunt tipped needle. The average amount of intragastric alcohol solution was about $0.45 \mathrm{~mL}$. The three ethanol binge were given consecutively each $12 \mathrm{~h}$ apart. Four hours after the last binge ethanol administration blood and liver samples were collected. Liver was perfused with cold phosphate buffered saline containing phosphatase inhibitors before collection. A small portion of the liver was placed in formalin for immunohistochemistry and the reminder was frozen in liquid nitrogen and stored at $-70{ }^{\circ} \mathrm{C}$. The animal care and protocol used in this study was approved by the University of Missouri Animal Care Committee (Protocol \#8175) and complied with NIH guide for the care and use of laboratory animals.

\subsection{Biochemical and Histological Studies}

Serum ALT levels were measured by kinetic ALT assay in an automated analyzer (University of Missouri Research Animal Diagnostic Laboratory, Columbia, MO, USA). The levels of Liver SAM, $\mathrm{SAH}$, adenosine and GSH were measured by high performance liquid chromatography as described earlier [11,30]. Formalin fixed liver tissue was embedded in paraffin, sectioned and stained with hematoxylin and eosin ( $\mathrm{H} \& \mathrm{E})$. Liver triglyceride levels were determined essentially as reported earlier by us [29]. Thirty $\mathrm{mg}$ of liver tissue was homogenized in $0.5 \mathrm{~mL}$ hypotonic buffer containing $20 \mathrm{mM}$ Tris, 2\% Triton X-100, and Sigma protease inhibitor cocktail (p8340) in a $2 \mathrm{~mL}$ capacity Dounce homogenizer with pestle A. The sample was heated to $60{ }^{\circ} \mathrm{C}$ followed by centrifugation at $13,000 \times g$ for $5 \mathrm{~min}$. The supernatant was used for triglyceride estimation using the assay kit and protocol provided by the supplier (Cat \#F6428, Sigma-Aldrich Company, St. Louis, MO, USA).

\subsection{Nuclear Isolation and Immunoblot Analysis}

The whole cell extract and nuclear extracts were prepared at $4{ }^{\circ} \mathrm{C}$ as described earlier [30]. After homogenizing the frozen liver tissue in lysis buffer (containing $50 \mathrm{mM}$ Tris. $\mathrm{HCl}$. pH 7.4, $25 \mathrm{mM} \mathrm{KCl}$, $5 \mathrm{mM} \mathrm{MgCl} 2,5 \mathrm{mM}$ glycerophosphate, $1 \mathrm{mM}$ EDTA, $1 \mathrm{mM}$ Na-orthovanadate, $1 \mathrm{mM}$ EGTA, $1 \mathrm{mM}$ DTT, and Sigma protease inhibitor cocktail (p8340)), a small portion of the whole cell extract was saved. The remainder of the homogenate was layered over 1.0 M sucrose cushion. After centrifugation at $1600 \times \mathrm{g}$ for $10 \mathrm{~min}$ at $4{ }^{\circ} \mathrm{C}$, the cytosolic fraction (supernatant) was transferred to a precooled microcentrifuge tube, frozen in liquid nitrogen and stored at $-70{ }^{\circ} \mathrm{C}$. The nuclear pellet was further suspended in $1.0 \mathrm{M}$ sucrose containing $0.25 \% \mathrm{NP}-40$ and passed through a 26-gauge needle 6 times and centrifuged at $1600 \times \mathrm{g}$ for $10 \mathrm{~min}$. This step removed contaminated endoplasmic reticulum and plasma membrane. The nuclear pellet was again re-suspended in $1.0 \mathrm{M}$ sucrose and centrifuged at $1600 \times g$ for 10 min followed by washing the pellet once with homogenization buffer. The purity of the nuclear fraction was verified by the absence of b-tubulin and calreticulin and presence of histone $\mathrm{H} 3$ [30]. The nuclear extract was prepared in high salt detergent buffer $(0.5 \mathrm{M} \mathrm{NaCl}, 1 \%$ Triton X-100, 
$1 \%$ deoxycholate and $0.1 \%$ SDS). Bio-Rad DC protein assay was used to determine the protein concentrations in whole cell extract and nuclear extract.

The whole liver lysate protein or nuclear fraction $(40 \mu \mathrm{g})$ was separated by $10 \%$ or $15 \%$ SDS-PAGE followed transfer onto nitrocellulose membrane using Bio-Rad Trans-Blot apparatus. Membrane was washed with $20 \mathrm{mM}$ Tris, $\mathrm{pH} 7.5$, containing $0.1 \%$ Tween 20 and $150 \mathrm{mM} \mathrm{NaCl}$ (TBST) and blocked with TBST containing 5\% nonfat dry milk for $2 \mathrm{~h}$ at room temperature. Membrane was incubated with primary antibody overnight at $4{ }^{\circ} \mathrm{C}$ in blocking buffer and washed with TBST, followed by incubation with corresponding secondary antibody conjugated with horseradish peroxidase for $1 \mathrm{~h}$ at room temperature. Immunoblots were visualized using chemiluminescent reagent (Pierce Chemicals, Rockford, IL, USA). LSA-3000 imaging system (Fujifilm Life Sciences, Stamford, Connecticut, USA) was used to capture chemiluminescence and Multi Gauge ${ }^{\mathrm{TM}}$ software (Fujifilm Life Sciences) was used for quantitation of immune blots. The chemiluminescence intensity was within the linear range of detection. Beta actin and histone $\mathrm{H} 3$ protein immune blots were used for equal protein loading of whole cell extracts and nuclear extracts respectively. Repeat immunoblotting was done by stripping the membrane using Restore Western blot stripping buffer (Pierce).

\subsection{Data Analysis}

Data obtained from 4 animals in each group were used for analysis. The data are expressed as mean \pm S.E.M. ANOVA was used for statistical analysis and differences with a $p$-value of $<0.05$ were considered statistically significant.

\section{Conclusions}

In conclusion, augmentation of liver injury by repeat acute binge in chronic ethanol treated mice is accompanied by increased levels of CYP2E1 protein, ALT, triglyceride, decrease in the SAM:SAH ratio, marked increase in hepatic adenosine levels, and increased H3 phosphorylation, a histone modification known to enhance TNF $\alpha$ gene expression. The association between gene polymorphism for CYP2E1, methionine metabolism, and TNF $\alpha$ has been considered in the alcoholic liver injury in humans [43]. Analogous changes observed in our acute on chronic model suggest that it may be a relevant experimental model for the study of progression of alcoholic liver injury. On the other hand, the increase in H2AX phosphorylation in binge but its suppression observed in the chronic ethanol binge group suggests that chronic ethanol intake antagonize the protective adaptive response of ethanol binge. The distinct pattern of hepatic methionine metabolism and epigenetic histone modifications but similar pattern of CYP2E1 protein induction in rat [30,33] and mouse (this study) models of ALD highlight common and distinct mechanisms of pathogenesis of liver damage. It may be tempting to suggest that the use of acute repeat binge on chronic ethanol intake for extended period of time may enhance alcoholic liver injury further mimicking the progression of liver injury in a setting commonly seen in humans.

\section{Author Contributions}

Conceived and designed experiments: Annayya R. Aroor, Shivendra D. Shukla; Performed experiments: Annayya R. Aroor, Shivendra D. Shukla, Ricardo Restrepo, Kusum K. Kharbanda; Analyzed data: 
Shivendra D. Shukla, Annayya R. Aroor, Ricardo Restrepo, Kusum K. Kharbanda and Jamal A. Ibdah; Wrote paper: Shivendra D. Shukla, Annayya R. Aroor, Ricardo Restrepo, Kusum K. Kharbanda and Jamal A. Ibdah.

\section{Conflicts of Interest}

The authors declare no conflict of interest.

\section{References}

1. Shukla, S.D.; Pruett, S.B.; Szabo, G.; Arteel, G.E. Binge ethanol and liver: New molecular developments. Alcohol Clin. Exp. Res. 2013, 37, 550-557.

2. Zakhari, S.; Li, T.K. Determinants of alcohol use and abuse: Impact of quantity and frequency patterns on liver disease. Hepatology 2007, 46, 2032-2039.

3. Rehm, J.; Mathers, C.; Popova, S.; Thavorncharoensap, M.; Teerawattananon, Y.; Patra, J. Global burden of disease and injury and economic cost attributable to alcohol use and alcohol-use disorders. Lancet 2009, 373, 2223-2233.

4. Mathurin, P.; Deltenre, P. Effect of binge drinking on the liver: An alarming public health issue? Gut 2009, 58, 613-617.

5. Grucza, R.A.; Norberg, K.E.; Bierut, L.J. Binge drinking among youths and young adults in the United States: 1979-2006. J. Am. Acad. Child Adolesc. Psychiatry 2009, 48, 692-702.

6. Courtney, K.E.; Polich, J. Binge drinking in young adults: Data, definitions, and determinants. Psychol. Bull. 2009, 135, 142-156.

7. Ceccanti, M.; Attili, A.; Balducci, G.; Attilia, F.; Giacomelli, S.; Rotondo, C.; Sasso, G.F.; Xirouchakis, E.; Attilia, M.L. Acute alcoholic hepatitis. J. Clin. Gastroenterol. 2006, 40, 833-841.

8. Mathurin, P.; Beuzin, F.; Louvet, A.; Carrié-Ganne, N.; Balian, A.; Trinchet, J.C.; Dalsoglio, D.; Prevot, S.; Naveau, S. Fibrosis progression occurs in a subgroup of heavy drinkers with typical histological features. Aliment. Pharmacol. Ther. 2007, 25, 1047-1054.

9. Crosse, K.I.; Anania, F.K. Alcoholic hepatitis. Curr. Treat Options Gastroenterol. 2002, 5, 417-423.

10. Zhu, H.; Jia, Z.; Misra, H.; Li, Y.R. Oxidative stress and redox signaling mechanisms of alcoholic liver disease: Updated experimental and clinical evidence. J. Dig. Dis. 2012, 13, 133-142.

11. Kharbanda, K.K. Methionine metabolic pathway in alcoholic liver injury. Curr. Opin. Clin. Nutr. Metab. Care 2013, 16, 89-95.

12. Peng, Z.; Borea, P.A.; Varani, K.; Wilder, T.; Yee, H.; Chiriboga, L.; Blackburn, M.R.; Azzena, G.; Resta, G.; Cronstein, B.N. Adenosine signaling contributes to ethanol-induced fatty liver in mice. J. Clin. Invest. 2009, 119, 582-594.

13. Peng, Z.; Fernandez, P.; Wilder, T.; Yee, H.; Chiriboga, L.; Chan, E.S.; Cronstein, B.N. Ecto-5'-nucleotidase (CD73)-mediated extracellular adenosine production plays a critical role in hepatic fibrosis. FASEB J. 2008, 22, 2263-2272.

14. Shukla, S.D.; Aroor, A.R. Epigenetic effects of ethanol on liver and gastrointestinal injury. World J. Gastroenterol. 2006, 12, 5265-5271.

15. Shukla, S.D.; Lim, R.W. Epigenetic effects of ethanol on the liver and gastrointestinal system. Alcohol Res. Curr. Rev. 2013, 35, 47-55. 
16. Patel, D.J.; Wang, Z. Readout of epigenetic modifications. Annu. Rev. Biochem. 2013, 82, 81-118.

17. Hübner, M.R.; Eckersley-Maslin, M.A.; Spector, D.L. Chromatin organization and transcriptional regulation. Curr. Opin. Genet Dev. 2013, 23, 89-95.

18. Pal-Bhadra, M.; Bhadra, U.; Jackson, D.E.; Mamatha, L.; Park, P.H.; Shukla, S.D. Distinct methylation patterns in histone H3 at Lys-4 and Lys-9 correlate with up- and down-regulation of genes by ethanol in hepatocytes. Life Sci. 2007, 81, 979-987.

19. Shukla, S.D.; Restrepo, R.; Fish, P.; Lim, R.W.; Ibdah, J.A. Different mechanisms for histone acetylation by ethanol and its metabolite acetate in rat primary hepatocytes. J. Pharmacol. Exp. Ther. 2015, 354, 18-23.

20. Kim, J.S.; Shukla, S.D. Acute in vivo effect of ethanol (binge drinking) on histone H3 modifications in rat tissues. Alcohol Alcohol. 2006, 41, 126-132.

21. Kirpich, I.; Zhang, J.; Gobejishvili, L.; Kharebava, G.; Barker, D.; Ghare, S.; Joshi-Barve, S.; McClain, C.J.; Barve, S. Binge ethanol-induced HDAC3 down-regulates Cpt1 $\alpha$ expression leading to hepatic steatosis and injury. Alcohol Clin. Exp. Res. 2013, 37, 1920-1929.

22. James, T.T.; Aroor, A.R.; Lim, R.W.; Shukla, S.D. Histone H3 phosphorylation (Ser10, Ser28) and phosphoacetylation (K9S10) are differentially associated with gene expression in liver of rats treated in vivo with acute ethanol. J. Pharmacol. Exp. Ther. 2012, 340, 237-247.

23. Aroor, A.R.; James, T.T.; Jackson, D.E.; Shukla, S.D. Differential changes in MAP kinases, histone modifications, and liver injury in rats acutely treated with ethanol. Alcohol Clin. Exp. Res. 2010, 34, 1543-1551.

24. Li, J.; Bardag-Gorce, F.; Oliva, J.; Dedes, J.; French, B.A.; French, S.W. Gene expression modifications in the liver caused by binge drinking and $S$-adenosylmethionine feeding. The role of epigenetic changes. Genes Nutr. 2010, 5, 169-179.

25. Esfandiari, F.; Medici, V.; Wong, D.H.; Jose, S.; Dolatshahi, M.; Quinlivan, E.; Dayal, S.; Lentz, S.R.; Tsukamoto, H.; Zhang, Y.H.; et al. Epigenetic regulation of hepatic endoplasmic reticulum stress pathways in the ethanol-fed cystathionine beta synthase-deficient mouse. Hepatology 2010, 51, 932-941.

26. Park, P.H.; Lim, R.W.; Shukla, S.D. Gene-selective histone H3 acetylation in the absence of increase in global histone acetylation in liver of rats chronically fed alcohol. Alcohol Alcohol. 2012, 47, 233-239.

27. Shukla, S.D.; Velazquez, J.; French, S.W.; Lu, S.C.; Ticku, M.K.; Zakhari, S. Emerging role of epigenetics in the actions of alcohol. Alcohol Clin. Exp. Res. 2008, 32, 1525-1534.

28. You, M.; Cao, Q.; Liang, X.; Ajmo, J.M.; Ness, G.C. Mammalian sirtuin 1 is involved in the protective action of dietary saturated fat against alcoholic fatty liver in mice. J. Nutr. 2008, 138, 497-501.

29. Aroor, A.R.; Jackson, D.E.; Shukla, S.D. Elevated activation of ERK1 and ERK2 accompany enhanced liver injury following alcohol binge in chronically ethanol-fed rats. Alcohol Clin. Exp. Res. 2011, 35, 2128-2138.

30. Aroor, A.R.; Restrepo, R.J.; Kharbanda, K.K.; Shukla, S.D. Epigenetic histone modifications in a clinically relevant rat model of chronic ethanol-binge-mediated liver injury. Hepatol. Int. 2014, $8,421-430$. 
31. Shinohara, M.; Ji, C.; Kaplowitz, N. Differences in betaine-homocysteine methyl transferase expression, endoplasmic reticulum stress response, and liver injury between alcohol-fed mice and rats. Hepatology 2010, 51, 796-805.

32. Arteel, G.E. Animal models of alcoholic liver disease. Dig. Dis. 2010, 28, 729-736.

33. Aroor, A.R.; Roy, L.J.; Restrepo, R.J.; Mooney, B.P.; Shukla, S.D. A proteomic analysis of liver after ethanol binge in chronically ethanol treated rats. Proteome Sci. 2012, doi:10.1186/1477-5956-10-29.

34. Lee, H.S.; Park, J.H.; Kim, S.J.; Kwon, S.J.; Kwon, J. A cooperative activation loop among $\mathrm{SWI} / \mathrm{SNF}$, gamma-H2AX and $\mathrm{H} 3$ acetylation for DNA double-strand break repair. EMBO J. 2010, 29, 1434-1445.

35. Szerlong, H.J.; Prenni, J.E.; Nyborg, J.K.; Hansen, J.C. Activator-dependent p300 acetylation of chromatin in vitro: Enhancement of transcription by disruption of repressive nucleosome-nucleosome interactions. J. Biol. Chem. 2010, 285, 31954-31964.

36. Choudhury, M.; Pandey, R.S.; Clemens, D.L.; Davis, J.W.; Lim, R.W.; Shukla, S.D. Knock down of GCN5 histone acetyltransferase by siRNA decreases ethanol-induced histone acetylation and affects differential expression of genes in human hepatoma cells. Alcohol 2011, 45, 311-324.

37. Schiltz, R.L.; Mizzen, C.A.; Vassilev, A.; Cook, R.G.; Allis, C.D.; Nakatani, Y. Overlapping but distinct patterns of histone acetylation by the human coactivators $\mathrm{p} 300$ and PCAF within nucleosomal substrates. J. Biol. Chem. 1999, 274, 1189-1192.

38. Aroor, A.R.; Jackson, D.E.; Shukla, S.D. Dysregulated phosphorylation and nuclear translocation of cyclic AMP response element binding protein (CREB) in rat liver after chronic ethanol binge. Eur. J. Pharmacol. 2012, 679, 101-108.

39. Iimuro, Y.; Bradford, B.U.; Yamashina, S.; Rusyn, I.; Nakagami, M.; Enomoto, N.; Kono, H.; Frey, W.; Forman, D.; Brenner, D.; et al. The glutathione precursor L-2-oxothiazolidine-4-carboxylic acid protects against liver injury due to chronic enteral ethanol exposure in the rat. Hepatology 2000, 31, 391-398.

40. Kharbanda, K.K.; Todero, S.L.; King, A.L.; Osna, N.A.; McVicker, B.L.; Tuma, D.J.; Wisecarver, J.L.; Bailey, S.M. Betaine treatment attenuates chronic ethanol-induced hepatic steatosis and alterations to the mitochondrial respiratory chain proteome. Int. J. Hepatol. 2012, doi:10.1155/2012/962183.

41. El Gazzar, M.; Yoza, B.K.; Chen, X.; Hu, J.; Hawkins, G.A.; McCall, C.E. G9a and HP1 couple histone and DNA methylation to TNF $\alpha$ transcription silencing during endotoxin tolerance. J. Biol. Chem. 2008, 283, 32198-32208.

42. Wang, B.; Chen, J.; Santiago, F.S.; Janes, M.; Kavurma, M.M.; Chong, B.H.; Pimanda, J.E.; Khachigian, L.M. Phosphorylation and acetylation of histone H3 and autoregulation by early growth response 1 mediate interleukin $1 \beta$ induction of early growth response 1 transcription. Arterioscler. Thromb. Vasc. Biol. 2010, 30, 536-545.

43. Brandon-Warner, E.; Schrum, L.W.; Schmidt, C.M.; McKillop, I.H. Rodent models of alcoholic liver disease: Of mice and men. Alcohol 2012, 46, 715-725.

(C) 2015 by the authors; licensee MDPI, Basel, Switzerland. This article is an open access article distributed under the terms and conditions of the Creative Commons Attribution license (http://creativecommons.org/licenses/by/4.0/). 